\title{
Mucking About in the Mess: Research-based Education at Quest University Canada
}

\begin{abstract}
The design of Quest University Canada began with a blank sheet of paper and a question: How do we create the most effective and engaging undergraduate education for students who will graduate into a rapidly changing, globalized world? The answer was to center education around the formulation of good questions and the processes by which one attempts to address them, rather than focusing instruction on the delivery of information. This leads naturally to having tutors who teach, rather than professors who profess. It leads to seminar rooms rather than lecture halls. It leads to dissolving disciplinary boundaries so good questions can easily cross them. It leads to a student-centered, project-based curriculum of exploration, rather than a fact-based transfer of information and its subsequent regurgitation. It leads to a collaborative rather than a competitive learning environment. And it produces students with highly developed skills in written and oral communication who are instinctively collaborative, inherently trans-disciplinary in their approach to problems, and engaged in their local and global communities.
\end{abstract}

Quest University Canada, the country's first independent, not-for-profit secular university, was created by an act of the British Columbia Legislative Assembly in 2002. By 2006, it had a faculty of seven and a program designed (and approved) to award a single degree: the Bachelor of Arts and Sciences. In September of 2007, we welcomed our first 73 students to a purpose-built campus in Squamish, British Columbia, between the city of Vancouver and the Olympic resort of Whistler. Today we have 50 faculty members, have reached our goal of 700 students, and have 250 graduates striving to change the world.

Most universities today are structured around a 19th-century model designed to produce graduates for a 19th-century economy-one that needed experts with heads crammed full of specialized information ready to be applied to an industrial enterprise. These experts usually had a single career during their working lives, and the pace of change in what they needed to know was modest. Problems were local or, at most, national in scale, and interaction with people from other cultures and traditions was unusual.

That world no longer exists. Vastly more information than could ever be stored in a single human brain is avail-

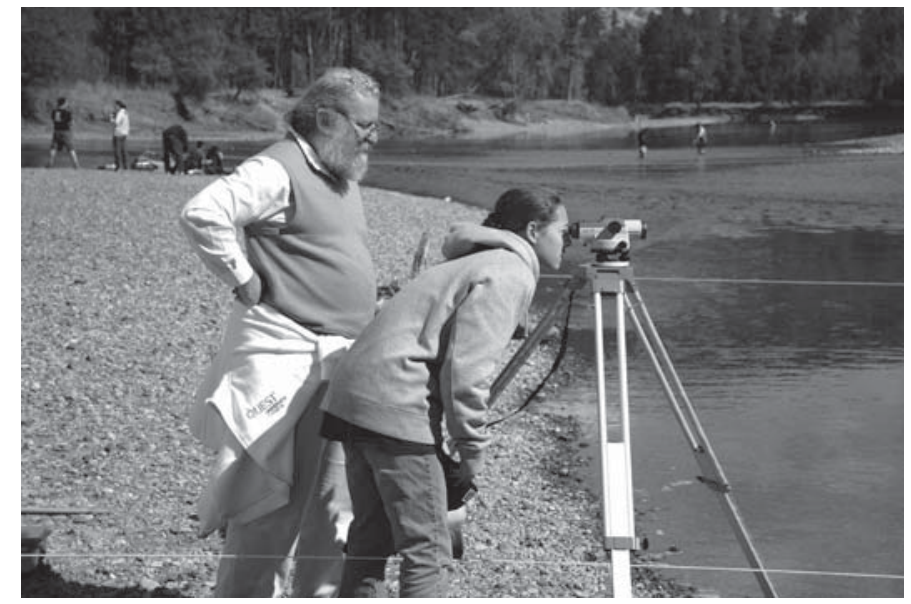

Author David Helfand helping a student profile a stream to assess its hydro power potential.

able in every pocket, a few swipes away on a smart phone. Manufacturing accounts for fewer than 13 million of the 149 million jobs in the United States (Lee and Mather 2008). The average university graduate today will have half a dozen different careers in his or her working lifetime, and half those careers don't exist yet. We create 2.5 quintillion bytes of new information per day (IBM 2015), making change endemic. And the major problems we face-water, food, trade, disease, climate-are all global in nature. We need a new model of education to prepare graduates for this new world.

\section{Designing a University}

In designing Quest, our first break with the past was to create a single faculty with no departments. We reinforced this ideal with concrete, by building a circular academic building with no boundaries and assigning faculty offices by lottery. As a result, a physicist sits next to a poet and on the other side has an economist, a botanist, and an expert in Chinese film and theatre. And they talk to each other, learn from each other, and work together to design a curriculum that transcends disciplinary silos.

Our campus has no lecture halls. Every classroom has a large oval table that can be easily sectioned into a variety of arrangements and around which only 21 chairs can fit. Thus all classes are capped at 20 students, and the average class size 


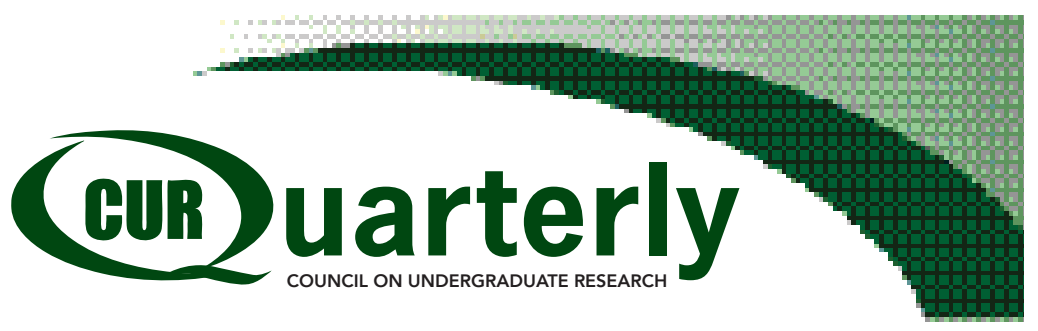

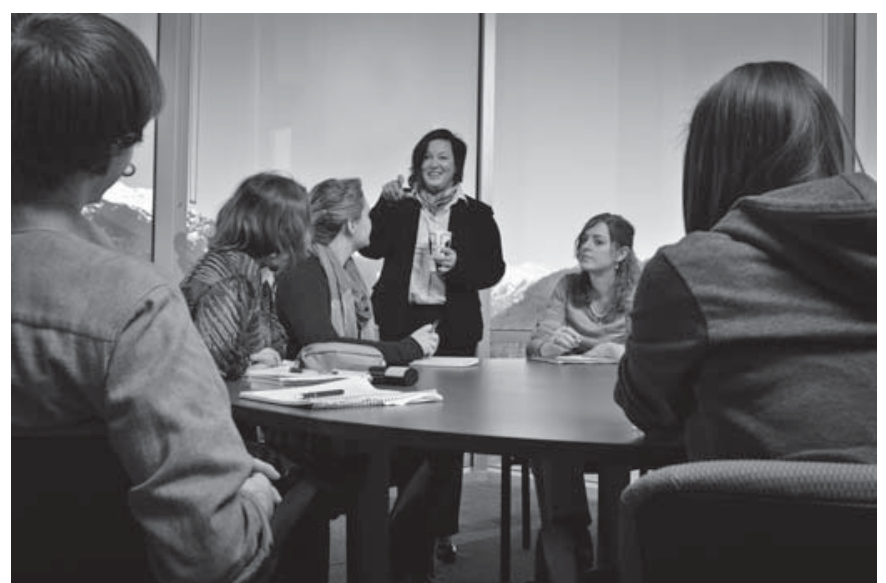

Psychology Tutor Dr. Megan Bulloch (pointing) in a seminar with her advisees (all full-time PhD faculty at Quest are called "Tutors").

is 16. Across from each classroom are small breakout rooms with four chairs, a table, and a whiteboard. A faculty member-called a tutor at Quest—can begin a class by setting up a problem, exploring a reading, or introducing an issue for debate. Then he or she can send the students off in small groups to attack different pieces of the problem, analyze the text, or draw up talking points before returning to the classroom. Other faculty members split the table into four pods of four or five students to analyze data or explore a computer simulation.

There are many other distinctions between Quest and traditional universities. For example, we have no faculty ranks. All tutors teach the same number of classes: three introductory and three advanced courses (six out of 12 blocks per year). We have no tenure; faculty members have multi-year contracts that are renewed (or not) after a review by an elected committee of their peers. All students live on campus all four years. Some faculty members live with their families in student residences and conduct evening programs there. Further, issues that arise in the residences often become fodder for classroom examination. We have intentionally constructed an integrated living and learning environment that includes students from 45 countries.

Our curriculum is also distinctive. We have a two-year "Foundation" program in which all students take the same 16 classes distributed across mathematics, physical sciences, life sciences, humanities, social sciences, arts, and language. Since we have no departments, we have no majors, so at the end of the two years, each student formulates a "Question" that will guide the last two years of his or her education. The construction of the Question requires: (1) finding a faculty mentor with whom the student will work one-on-one for the next two years; (2) selecting a set of touchstone readings to gain an understanding of the foundations of the disciplinary fields on which the Question focuses; (3) planning a set of classes to enroll in (from among the courses in the "Concentration" track that follows the Foundation courses); (4) devising an experiential-learning plan of from one to four months off-campus in a work or research setting; and, finally, (5) creating a "Keystone" project that summarizes what the student has learned by pursuing the question. The Keystone project is presented to the entire university community on the last days of the final term and is archived in the university library.

While both the philosophy and the structure of this curriculum envision a problem-based, research-oriented approach to learning, the key feature of our design that enables this to be effective is the "block" system. Block scheduling means students take four courses in a term, but they take them sequentially rather than simultaneously. Each block lasts for 24 days. Classes nominally meet three hours a day (9-12 or 1-4) five days a week for a total of 54 contact hours, more than a typical semester-long course. The expectation is that students will, on average, do five hours of work a day outside of class, and both students' self-reports and a review of the work accomplished confirm that this expectation is being met. The level of students' engagement and the depth of understanding they can reach in this immersive system is well beyond that attainable with standard semester-based calendars. Furthermore, this schedule is ideally suited to research-based education.

Research, almost by definition, cannot be conducted in a time-constrained manner. Exploration doesn't have a pre-set schedule, and the task of drawing conclusions from a collection of information is an activity that can rarely be tied to the clock. At Quest, the block system abolishes time constraints. A field trip need not be completed by noon because a student has a lab class that afternoon. An unusually large assignment will not conflict with a student's English paper due the next day. Thus, field trips can last a day, a week, or the entire month. Assignments can be open-ended without compromising a student's work in other classes. The student and the faculty member have just one thing to focus on, and the only time horizon is the start of the next block four weeks away.

\section{The Foundation Program}

Each 200-student entering cohort begins the Quest program in one of 10 sections of the "Cornerstone" class. This course serves several purposes: to introduce students to the rhythm of work on the block schedule, to administer diagnostics and 


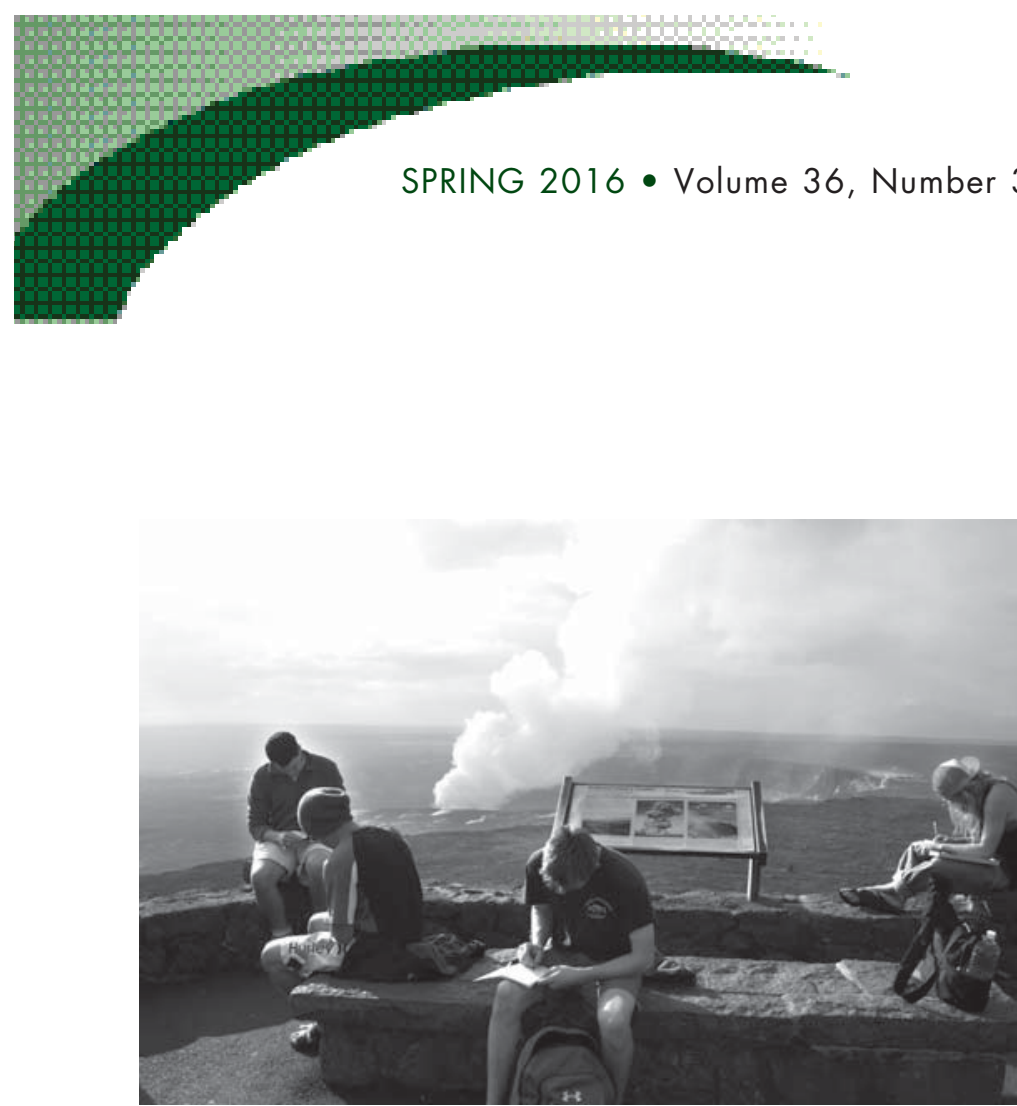

Geology tutor Steve Quane (left) with students at the Volcano National Observatory in Hawaii.

set the bar for the level of work expected in both writing and quantitative reasoning, to illustrate community norms and expectations, to facilitate students' introduction to group discussion and collaborative work, and to introduce the idea of a process-based, rather than the familiar answer-based, educational model.

The Cornerstone class is highly multidisciplinary. It explores the question "What is Knowledge?" from the point of view of anthropology, ancient and modern philosophy, literature, art, mathematics, and science. The science component provides an immediate introduction to our research-based approach. On the second Friday of the block, students are presented with 60 research questions that have been developed by the 20 faculty members teaching the class. These questions can all be pursued within the boundaries of a partially wooded area on the campus. Examples include:

- Does tree growth correlate with precipitation levels over the past three decades?

- Does stomatal density on leaves vary with height on a tree?

- Are there diurnal variations in leaf water content?

- How many fungal species exist in the study area?

- What do humans use to navigate in a new, unfamiliar area?

The students form themselves into groups of three or four (often across sections of the class), and each team chooses a question. They are given access to lab and field equipment, and some minimal guidance by the faculty member responsible for their question. They have from Friday until Tuesday at noon to complete an exploration of their question and to prepare a poster presentation for a conference that opens after lunch that day.

As an example, the group pursuing the first question above must learn to use a Swedish borer to get core samples from several trees, develop a way to quantify the widths of the trees' annual growth rings, find the historical precipitation records for the area, and calculate the correlation between rainfall and ring width. The group attacking the third question needs to collect leaves over one or more 24-hour periods and use a laboratory oven and a balance to assess water content as a function of time. The group choosing the fifth question has to design an experiment and find volunteers to participate in determining how people orient themselves in a forest. All groups learn about experimental procedures, measurement uncertainties, data presentation, and, in many cases for the first time, both the value and limitations of scientific knowledge. As one participant said to me with genuine delight, "I hated the science we did in high school. I had no idea this is what science is."

A number of the other Foundation courses that follow the Cornerstone course are designed to spend a substantial fraction of class time in the field. The three life-science courses are focused on the ecosystem, organismal, and cellular/ molecular levels, respectively, and the ecosystem course is titled "The Biodiversity of British Columbia." Students spend at least every other day in the field, rain or shine. We are fortunate to have ecosystems ranging from intertidal zones to alpine meadows all within an hour of campus. Whether by van or on foot, the students are out doing measurements, collecting samples to examine in the lab, and addressing assignments phrased as research questions rather than as problem sets. Students in the physical science course, "Earth, Oceans \& Space," also spend a large amount of time using water-sampling equipment and rock hammers to explore the local environment.

Field trips are not limited to science classes. The flexibility of block scheduling allows a class on world religions to attend a Buddhist retreat in the evening when it is open and a synagogue on Saturday morning. The penultimate day in the Mandarin language class sees the students deposited in Richmond, British Columbia, where many residents are Chinese and all the signage and stores and restaurant names are in Chinese characters. They are given a set of tasks to accomplish and celebrate their completion with a dim sum meal (ordered in Mandarin, of course).

Not all subjects lend themselves to hands-on research projects in an undergraduate class. Nonetheless, it is often possible to create inquiry-based activities that mimic the processes 
party how someone else solved a problem and then asked to replicate that solution. This can develop technical competence, but it doesn't lead either to innovation or mastery. Creating a culture of inquiry-a culture in which "mucking about in the mess" is celebrated-is the key to an engaging curriculum that produces first-person learning in graduates eager to embrace the challenges they will face in this new millennium.

\section{Evaluation}

In 2005, the American Association of Colleges and Universities launched the LEAP project (Liberal Education and America's Promise). A central part of the agenda was to define what it called "high impact practices"—activities that research has validated as enhancing student outcomes. The resulting comprehensive list informed our design at Quest: First-year experiences (our Cornerstone class), learning communities (set up in the Cornerstone), writing-intensive courses (every student's second block is rhetoric), collaborative assignments and projects (every class features these), undergraduate research (required of all students), diversity/global learning (80 percent of our graduates have been abroad as part of their Quest education), service learning (required in some classes), internships (required of all students), and capstone courses and projects (the Keystone project and final block required of all students) (Kuh 2008).

We use two standardized instruments, plus a number of locally generated ones, to measure the effectiveness of this educational approach. One measure used is the National Survey of Student Engagement (NSSE, http://nsse.indiana.edu), a 15-year-old instrument used by more than 2,000 North American colleges and universities. It includes more than 100 questions probing what students are doing (e.g., "How many hours a week do you spend discussing academic work with your fellow students outside of class?") and develops 10 measures of excellence in undergraduate education. For five years in a row, Quest has been the number one university in Canada on all 10 measures, for both first- and fourth-year students. And in the last year in which U.S. results were available, we were number one in North America.

The Collegiate Learning Assessment is a newer instrument designed to measure the value added by a university education. It compares students' expected academic performance based on an entrance test to the results of an exit exam upon their graduation. It solicits written responses to situational prompts requiring quantitative and qualitative analysis, as well as persuasive writing. In our first two years of using this instrument, we are in the top 10 percent of North American institutions, and 30 percent of our graduating students scored in the 99 th percentile.

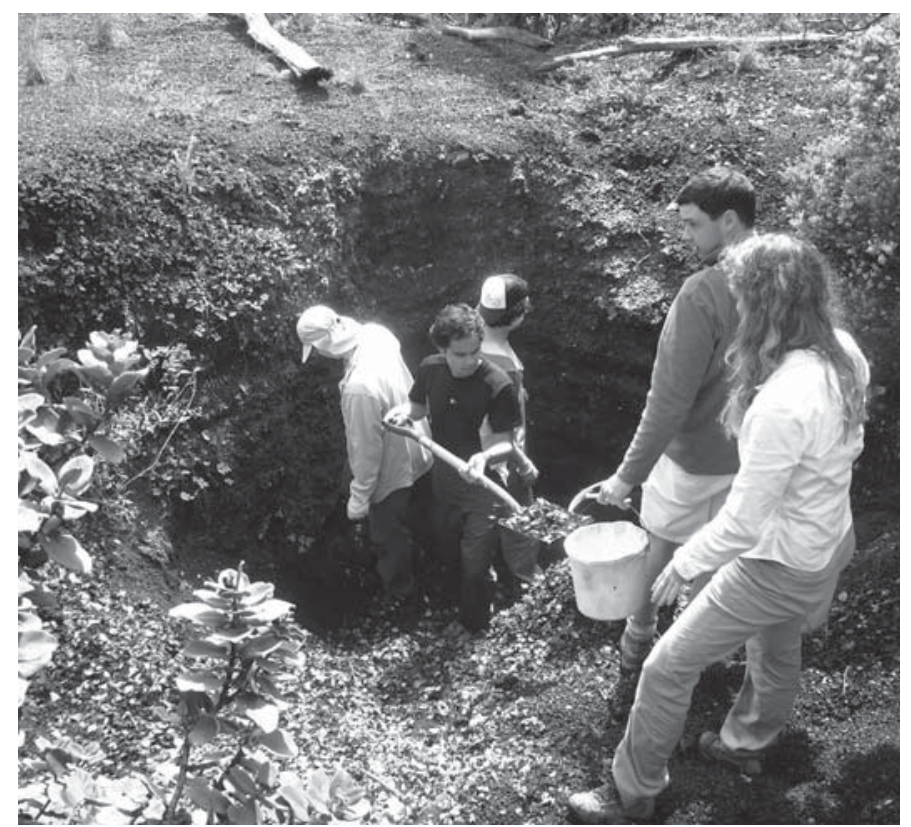

Geology Tutor Steve Quane (second from right) with his students excavating a lava flow.

We have also developed a comprehensive quantitative-reasoning program that includes entrance and exit diagnostics and mandatory tutoring for students lacking the level of skills required as prerequisites for particular classes. Similar diagnostics exist in our rhetoric/writing-across-the-curriculum program. Our ultimate outcomes assessment, however, is the success our students achieve after graduation, when they excel in leading graduate and professional programs around the world and thrive in a wide variety of occupations.

\section{Wider Adoption}

Part of the original Quest vision was not only to create a uniquely effective model of undergraduate education, but also to influence the future of higher education in general. In just eight years, this secondary goal is already being attained. After studying our program for three years, Hearst University, a public institution in Ontario, completely switched to our block system last September. The faculty at Augustana College, a branch of the University of Alberta, voted in May 2015 to implement a block-scheduling system in 2017, and individual programs at several other Canadian universities and colleges have adopted the block approach to curriculum. The first new university to be established in the UK in 35 years (NMiTE in Hereford was announced in February 2015 and says in its founding documents that it was "inspired by Quest University Canada." Further, the Aga Khan Development Network is building a new universi- 
ty in Arusha, Tanzania, based on the Quest model. And the academic leading the Conference Board of Canada's current study of postsecondary education, Carl Amrhein, has said, "We should put a Quest in every major population centre in Canada and see how the public institutions respond to the competition."

Adoption of the block schedule is inhibited primarily by institutional and faculty inertia. It is not inherently more costly and, indeed, it can lead to institutional efficiencies such as well-subscribed summer academic blocks and the sharing of offices by faculty who teach during alternate blocks. It does, however, require faculty members to completely re-think course delivery. It is simply untenable to stand and lecture from PowerPoint slides for three hours straight every day of the week and expect an attentive audience. But such rethinking would be a highly salutary pedagogical outcome.

While it is not possible for students to participate in typical semester-long courses and in our type of academic blocks simultaneously, any school, program, or major can operate on the block schedule if the student cohort is confined to that schedule. For example, geography majors at the University of Northern British Columbia now study on the block system in their third and fourth years while the rest of the institution operates on the old semester system.

While a limit of 20 students in a class may not be easy to achieve at many large universities, it is worth noting that the total operating cost per student at Quest is lower than that for the average Canadian public university. Further, it is possible to introduce active learning into larger classes. For example, there is no reason (beyond, perhaps, the configuration of classroom furniture) that a class of 200 students could not carry out the Kepler's Laws exercise described above in groups of five as did my class of 20 students. My role in the class (beyond the substantial work of designing the exercise) was simply to be there as a resource to answer questions, something a small cadre of teaching assistants could do in a scaled-up version of the class.

Another central tenet of our system costs absolutely nothing to implement: the substitution of an ethos of collaboration for the current competitive atmosphere induced by grading-on-a-curve. A classroom suffused by peer learning is highly effective, inherently motivating, and a better model of real world workplaces than an atmosphere of individualistic competitive behavior that increases stress and promotes rampant cheating. Collaboration is especially valuable in a research-based curriculum, where the multiple strengths required to solve a problem can be drawn from different participants.

In most major research universities, the opportunity for senior undergraduates to participate in genuine research projects is restricted to a small fraction of honors students. The implementation of a universal "Question" plan and "Keystone" project would require some reorientation of faculty members' priorities from their own research to the mentoring of undergraduates. However, at Princeton University, a senior thesis is a graduation requirement, proof that this is possible at research-intensive institutions.

\section{Conclusions}

A research-based academic model is messy, inefficient, and time-consuming. It celebrates questions over answers, so students' work is less easy to grade. It requires the faculty member to step off the podium and engage with students. And it requires students to develop collaboration and communication skills, creativity, resilience in the face of failure, and a willingness to question - all attributes that lead to success in the $21^{\text {st }}$ century economy.

\section{References}

Derby-Talbot, Ryan. 2013. "Cats on Ladders and First-Person Learning Experiences; Ryan Derby-Talbot at TEDxSquamish." YouTube video, 16:06, posted by TEDx Talks, November 26, 2013, Accessed September 14, 2015. https://www.youtube.com/watch?v=-tFCVa3lCds.

IBM. 2015. "What is Big Data?" Accessed September 14, 2015. www-01.ibm. $\mathrm{com} /$ software/data/bigdata/what-is-big-data.html.

Kuh, George D. 2008. High-Impact Educational Practices: What They Are, Who Has Access to Them, and Why They Matter. Washington DC: Association of American Colleges and Universities. Accessed 14 September 2015 http:// www.aacu.org/resources/high-impact-practices.

Lee, Marlene A. and Mark Mather. 2008. US Labor Force Trends. Population Bulletin: A Publication of the Population Reference Bureau 63(2): 2008.

\section{David J. Helfand}

Quest University Canada, david.helfand@questu.ca

David J. Helfand was president and vice chancellor of Quest University Canada from 2008 to August 2015 He has been a faculty member at Columbia University for 39 years; half of that time he served as chair of the Department of Astronomy. He is the author of nearly 200 scientific publications and has mentored 22 $P h D$ students, but most of his pedagogical efforts have been aimed at teaching science to non-science majors. He instituted the first change in Columbia's Core Curriculum in 50 years by introducing science to all first-year students. In 2005, he helped create Quest, Canada's first independent, non-profit, secular university. He recently completed his final year of a four-year term as president of the American Astronomical Society. His first book, A Survival Guide to the Misinformation Age, will appear this winter.

doi: $10.18833 /$ curq/36/3/4 